\title{
A revised solution for a sphere rolling in a vertical loop
}

\author{
Oscar Bertran* \\ Department of Physics, EETAC, UPC \\ Jordi Riba \\ Department of Electrical Engineering, ESEIAAT UPC
}

(Dated: September 15, 2020)

\begin{abstract}
In this paper the classical well known solution of the elementary problem of the sphere loop in the presence of friction is studied. Also, a modification of the problem shows a possible inaccuracy involved on the well known solution found in many references. Finally a revised solution of the problem is presented.
\end{abstract}




\section{INTRODUCTION}

One classical problem in elementary physics is the analysis of a particle rolling ang slipping on an inclined plane, which has been deeply analyzed in the literature. ${ }^{1}$ Shaw and Wunderlick ${ }^{2}$ performed a deep analysis of the slipping and rolling motion of a sphere in an inclined track based on experimental data.

Another classical problem in elementary physics found in many text books consists on finding the minimum height needed for an object starting from rest, that slides down an inclined plane, either frictionless or with friction, to reach the top of a frictionless vertical circular loop. This problem and diverse variants have been widely studied in the literature. ${ }^{3-6}$

This paper develops a revised solution for the classical problem of determining the minimum initial height for a sphere that starts from rest, and rolls down an inclined plane, to complete a vertical circular loop in the presence of friction. It is proved that the conventional solution found in many text books has several flaws, which are discussed and compared with the solution presented in this paper. Although a similar problem has been already studied in the literature ${ }^{7}$, in this work, Tea analyzes different particular cases focusing the attention around a reference initial condition $h / R=2.7, h$ being the initial height of the sphere, and $R$ the radius of the vertical loop, but searching for a general solution of this problem is not the focus of Tea's paper.

The paper is organized as follows. Section II presents the classic problem and the conventional solution. Section III modifies the classical problem and the proposed solution, highlighting the improvements with respect to the conventional solution provided in physics text books. Section IV describes the complete solution of the problem. Section V discuses the different possible solutions. Section VI develops the comments and conclusions.

\section{THE CONVENTIONAL SOLUTION}

The conventional problem of the sphere loop states: "Determine the minimum initial height for a sphere of mass $m$ and radius $r$, starting from rest, and rolling down an inclined plane, to complete a vertical circular loop of radius $R$ in the presence of friction assuming only one contact point between the sphere and the loop."

The conventional solution found in physics text books involves an inclined plane where 
the sphere rolls down without slipping till it initiates the loop. This is not the most important part of the motion because the inclined plane can have a very small angle ensuring a perfect rotation of the sphere along the inclined plane. By applying the energy conservation principle, the following expression can be easily derived,

$$
m g h=\frac{1}{2} m v_{0}^{2}+\frac{1}{2} I \omega_{0}^{2}
$$

where $h$ is the initial height of the sphere above the bottom point of the vertical loop, $v_{0}$ and $\omega_{0}$ are, respectively, the linear and angular velocities of the sphere when entering the loop, $I$ the moment of inertia of the sphere and $g$ the gravitational acceleration. By substituting $\omega_{0}=v_{0} / r$ and $I=\frac{2}{5} m r^{2}$ in Eq. (1), it results in,

$$
h=\frac{7 v_{0}^{2}}{10 g}
$$

Next, the conventional solution imposes two conditions (Section III demonstrates the unfeasibility of applying both impositions simultaneously):

1. First condition: to complete the loop, the minimum velocity of the sphere entering the vertical loop at the bottom point, is that allowing the sphere to reach the top of the loop at the exact velocity, so that the normal force at this point is zero. This condition is well formulated. The top point of the circular loop is the most critical point to ensure a contact between the sphere and the track. The top point of the loop accomplishes,

$$
F_{N}+m g=m \frac{v^{2}}{R-r}
$$

By supposing that the normal force is zero at the top point of the loop, Eq. (3) results in,

$$
v^{2}=g(R-r)
$$

Usually, most of the solutions assume that the radius of the sphere is very small compared with the radius of the loop $(r<<R)$, so Eq. (4) results in,

$$
v^{2}=g R
$$




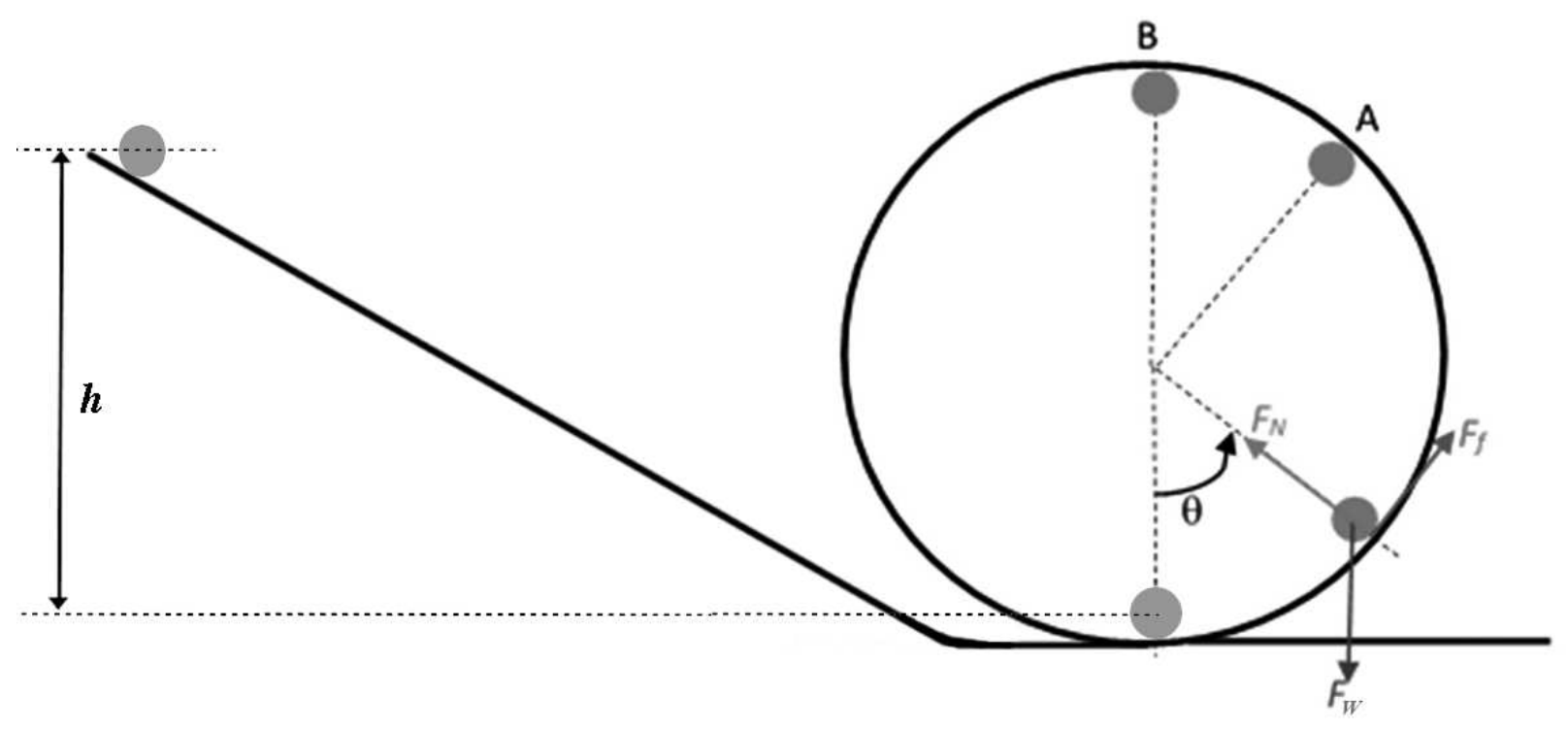

FIG. 1. Vertical circular loop diagram

2. Second condition: the sphere will complete the loop following a perfect rotational motion in all points of the trajectory, i.e., without slipping. This condition ensures the conservation of energy, so Eq. (6) can be applied between the bottom and the top point of the loop,

$$
\frac{1}{2} m v_{0}^{2}+\frac{1}{2} I \omega_{0}^{2}=m g 2(R-r)+\frac{1}{2} m v^{2}+\frac{1}{2} I \omega^{2}
$$

By assuming $r<<R$, using the moment of inertia of the sphere $\left(I=\frac{2}{5} m r^{2}\right)$ and supposing the sphere rolls without slipping $(v=r \omega)$, Eq. (6) results in,

$$
v_{0}^{2}=\frac{20}{7} g R+v^{2}
$$

Substituting Eq. (5) in Eq. (7) the resulting linear velocity at the bottom of the loop results in,

$$
v_{0}^{2}=\frac{27}{7} g R
$$

This is the minimum velocity of the sphere to complete the loop, being always in 
contact with the loop track.

So, from Eq. (2), the minimum initial height of the sphere is:

$$
h=\frac{7 v_{0}^{2}}{10 g}=2.7 R
$$

The solution in Eq. (9) is well-known and the most accepted result of the problem, although some issues arise as discussed in Section IV

\section{MODIFYING THE USUAL PROBLEM}

This problem can be analyzed by means of a force balance approach. Figure 1 shows the forces applied to the sphere. By applying the second Newton's law the following expressions can be easily derived,

$$
\begin{aligned}
F_{f}-m g \sin (\theta) & =m a_{t} \\
F_{N}-m g \cos (\theta) & =m \frac{v^{2}}{R-r}
\end{aligned}
$$

Equation (10) refers to the force balance along the tangent axis, whereas Eq. (11) describes the forces along the radial axis. By analyzing the rotational motion of the sphere, the following equations arise,

$$
\begin{aligned}
-r F_{f} & =I \alpha=\frac{2}{5} m r^{2} \alpha \\
F_{f} & =-\frac{2}{5} m r \alpha
\end{aligned}
$$

$F_{f}$ being the friction force, $m$ the mass of the sphere, $g$ the gravitational acceleration, $a_{t}$ the tangential component of the acceleration, $F_{N}$ the normal force, $v$ the linear velocity of the center of the sphere and $\alpha$ the angular acceleration of the sphere about the axis that passes through the center of mass perpendicularly to the plane of the motion.

Assuming that the sphere rolls without slipping, the friction force must be replaced by a static friction force in all points of the loop path, where,

$$
F_{f s} \leq \mu_{s} F_{N}
$$


At this point an issue arises. According to the conventional solution, the normal force at the top of the loop is zero, so in all points of the loop path, the static friction force must be less than a maximum value $\mu_{s} F_{N}$, which tends to zero when approaching to the top of the loop, this being a weakness of the conventional solution.

To solve this issue, the conventional problem can be modified according to the following statement: "Determine the minimum initial height for a sphere of mass $m$ and radius $r$, starting from rest, and rolling down an inclined plane, to complete a vertical circular loop of radius $R$ in the presence of friction, while ensuring that it completes the loop without slipping in all points of the loop path assuming only one contact point between the sphere and the loop". The aim of this modified statement is to obtain a result which is independent of the static friction coefficient, or at least allowing to limit the value of $\mu_{s}$ while providing a solution for the velocity equal to that found by means of the conventional solution.

This problem can be solved by applying rotational motion without slipping of the sphere $\left(a_{t}=r \alpha, v=r \omega\right)$, Eq. (13) is transformed as,

$$
F_{f s}=-\frac{2}{5} m a_{t}
$$

And from Eqs. (10) and (15) it results,

$$
F_{f s}=\frac{2}{7} m g \sin (\theta)
$$

The energy conservation equation applied in all points of the loop results in,

$$
\frac{1}{2} m v_{0}^{2}+\frac{1}{2} I \omega_{0}^{2}=m g(R-r)(1-\cos (\theta))+\frac{1}{2} m v^{2}+\frac{1}{2} I \omega^{2}
$$

Which can be applied for all values of $\theta$ in the loop. By assuming that the sphere rolls without slipping, Eq. (17) results in,

$$
v^{2}=v_{0}^{2}-\frac{10}{7} g(R-r)(1-\cos (\theta))
$$

By substituting Eq. (18) in Eq. (11),

$$
F_{N}=m\left(g\left(-\frac{10}{7}+\frac{17}{7} \cos (\theta)\right)+\frac{v_{0}^{2}}{R-r}\right)
$$


From Eq. (14) and Eq. (16),

$$
\frac{2}{7} m g \sin (\theta) \leq \mu_{s} m\left(g\left(-\frac{10}{7}+\frac{17}{7} \cos (\theta)\right)+\frac{v_{0}^{2}}{R-r}\right)
$$

Next, by solving Eq. (20) for $v_{0}$,

$$
v_{0}^{2} \geq \frac{g(R-r)}{7}\left(10-17 \cos (\theta)+\frac{2 \sin (\theta)}{\mu_{s}}\right)
$$

And by approximating $r<<R$ :

$$
v_{0}^{2} \geq \frac{g R}{7}\left(10-17 \cos (\theta)+\frac{2 \sin (\theta)}{\mu_{s}}\right)
$$

According to Eq. (22), the value of the square of the velocity at the bottom point of the loop must be greater or equal than any value of the function for $\theta=[0,2 \pi] \mathrm{rad}$,

$$
F(\theta)=10-17 \cos (\theta)+\frac{2 \sin (\theta)}{\mu_{s}}
$$

The maximum of function $F(\theta)$ results in,

$$
\tan \left(\theta_{M}\right)=\frac{-2}{17 \mu_{s}}
$$

This angle is placed in the second or fourth quadrant,, but the feasible solution is that of the second quadrant $\left(\frac{\pi}{2}<\theta_{M}<\pi\right)$, so by applying the following trigonometric identities,

$$
\cos \left(\theta_{M}\right)=\frac{-1}{\sqrt{1+\tan ^{2}\left(\theta_{M}\right)}} \quad \sin \left(\theta_{M}\right)=\frac{-\tan \left(\theta_{M}\right)}{\sqrt{1+\tan ^{2}\left(\theta_{M}\right)}}
$$

in Eq. (22) results in,

$$
v_{o}^{2} \geq \frac{g R}{7}\left(10+\frac{17}{\sqrt{1+\tan ^{2}\left(\theta_{M}\right)}}-\frac{2 \tan \left(\theta_{M}\right)}{\mu_{s} \sqrt{1+\tan ^{2}\left(\theta_{M}\right)}}\right)
$$

By substituting Eq. (24) into Eq. (26),

$$
v_{0}^{2} \geq \frac{g R}{7}\left(10+17 \sqrt{1+\left(\frac{2}{17 \mu_{s}}\right)^{2}}\right)
$$


and by substituting Eq. (9) into Eq. (27),

$$
h \geq \frac{R}{10}\left(10+17 \sqrt{1+\left(\frac{2}{17 \mu_{s}}\right)^{2}}\right)
$$

It is easy to prove that with the minimum velocity given by Eq. (27), the normal force at the upper point of the loop is not zero and therefore, this value is not the minimum velocity at the bottom of the loop to reach the upper point assuming that the sphere rolls without slipping. This value is higher than the accepted one for the conventional solution. When using an infinite static friction coefficient in Eq. (27) or Eq. (28), the $v_{0}$ and $h$ values of the conventional solution are obtained. It can be concluded that when the velocity $v_{0}$ is below the threshold value in Eq. (27), the sphere cannot complete the vertical loop rolling without slipping in all points of the path. Following another formulation, Tea ${ }^{7}$ also concludes that according to the conventional solution of the classical problem ( $h=2.7 R$ ), the static friction coeffcient must tend to infinite.

\section{SOLVING THE CLASSICAL PROBLEM}

In the absence of any force other than the weight and the normal force, when a solid body is forced to follow a vertical loop trajectory, as the height of the body increases the value of the normal force decreases. At the point where the normal force is zero, the body separates from the loop track. Therefore, in all cases, the minimum initial height or the minimum speed at the lowest point of the trajectory to complete the loop is found by solving the limit case, i.e., when the normal force is zero at the highest point of the loop.

Results presented in Section III prove that in order to complete the loop (rolling with or without slipping), the minimum velocity $v_{0}$ of the sphere at the bottom point, is lower than the minimum velocity provided by (27) in Section III (minimum speed to complete the loop rolling without slipping).

Assume a sphere that launched with unknown linear velocity $v_{0}$ at the bottom point of the vertical loop, rolling without slipping until point $A$ (see Fig. 1)). From $A$, the sphere slips until reaching point $B$, where the normal force is zero, as detailed in Section II. It is noted that from the lowest point until point $A$, a static friction force must be taken into account, whereas after point $A$ a dynamic friction force is considered. 
The velocity of the center of the sphere in point $B$ is $v_{B}^{2}=g R$. This is true under the assumption that the normal force is zero in point $B$, and by assuming that $r<<R$. Equations (10), (11) and (13) describe the motion from $A$ to $B$. In this case, the friction force must be considered a dynamic friction force, thus obtaining,

$$
\begin{aligned}
F_{f d}-m g \sin (\theta) & =m a_{t} \\
F_{N}-m g \cos (\theta) & =m \frac{v^{2}}{R} \\
F_{f d} & =-\frac{2}{5} m r \alpha
\end{aligned}
$$

In this case, the sphere rolls and slips $\left(a_{t} \neq r \alpha\right)$, but since the friction force is given by $F_{f d}=\mu_{d} F_{N}$

From Eq. (11), under the assumption $r<<R$,

$$
F_{N}=m\left(g \cos (\theta)+\frac{v^{2}}{R}\right)
$$

Since the friction force is givem by $F_{f d}=\mu_{d} F_{N}$, Eq. (29) results in,

$$
\mu_{d} m\left(g \cos (\theta)+\frac{v^{2}}{R}\right)-m g \sin (\theta)=m a_{t}
$$

Equation (33) can be rewritten as,

$$
a_{t}-\mu_{d} \frac{v^{2}}{R}=g\left(\mu_{d} \cos (\theta)-\sin (\theta)\right)
$$

There are two ways to solve Eq. (34), by means of the Volterra's approach and the solution explained in the following lines. Since a circular motion is analyzed, the following kinematic definitions can be applied,

$$
a_{t}=\frac{d v}{d t} \quad v=\frac{d s}{d t}
$$

Where $d s$ is the differential arc of the circumference $(d s=R d \theta)$, so it results,

$$
\frac{d v}{a_{t}}=\frac{d s}{v} \quad v d v=a_{t} d s \quad d v^{2}=2 v d v
$$


or, $d v^{2}=2 a_{t} R d \theta$

$$
a_{t}=\frac{1}{2 R} \frac{d v^{2}}{d \theta}
$$

Next, by substituting Eq. (37) into Eq. (34),

$$
\frac{d v^{2}}{d \theta}-2 \mu_{d} v^{2}=2 g R\left(\mu_{d} \cos (\theta)-\sin (\theta)\right)
$$

The solution of Eq. (38) results in,

$$
v^{2}=g R\left(\frac{2\left(1-2 \mu_{d}^{2}\right)}{1+4 \mu_{d}^{2}} \cos (\theta)+\frac{6 \mu_{d}}{1+4 \mu_{d}^{2}} \sin (\theta)\right)+C \exp \left(2 \mu_{d} \theta\right)
$$

where $C$ is a constant that depends on the initial or boundary values. In the analysed case, since the angular position of $B$ is $\theta_{B}=\pi$, the normal force is 0 , so, $v_{B}^{2}=g R$ :

$$
\begin{aligned}
g R & =g R\left(-\frac{2\left(1-2 \mu_{d}^{2}\right)}{1+4 \mu_{d}^{2}}\right)+C \exp \left(2 \mu_{d} \pi\right) \\
C & =\begin{array}{c}
3 g R \\
1+4 \mu_{d}^{2}
\end{array}
\end{aligned}
$$

thus Eq. (39) results in,

$$
v^{2}=\frac{g R}{1+4 \mu_{d}^{2}}\left(6 \mu_{d} \sin (\theta)+2\left(1-2 \mu_{d}^{2}\right) \cos (\theta)+3 \exp \left(2 \mu_{d}(\theta-\pi)\right)\right)
$$

It is noted that (42) is the expression of the square of the velocity of the sphere center between points $A$ and $B$. The velocity in point $A$ can be expressed as,

$$
v_{A}^{2}=\frac{g R}{1+4 \mu_{d}^{2}}\left(6 \mu_{d} \sin \left(\theta_{A}\right)+2\left(1-2 \mu_{d}^{2}\right) \cos \left(\theta_{A}\right)+3 \exp \left(2 \mu_{d}\left(\theta_{A}-\pi\right)\right)\right)
$$

Since point $A$ is the highest point at which the sphere rolls without slipping, from $A$ to $B$ the sphere slips, and thus the limit of the static friction force must be applied at point $A$, so, $F_{s A}=\mu_{s} N_{A}$

From Eq. (16) and Eq. (20)

$$
\frac{2}{7} m g \sin \left(\theta_{A}\right)=\mu_{s} m\left(g \cos \left(\theta_{A}\right)+\frac{v_{A}^{2}}{R}\right)
$$

By substituting Eq. (44) in Eq. (43) it results, 


$$
3 \cos \left(\theta_{A}\right)+\frac{42 \mu_{d} \mu_{s}-8 \mu_{d}^{2}-2}{7 \mu_{s}} \sin \left(\theta_{A}\right)+3 \exp \left(2 \mu_{d}\left(\theta_{A}-\pi\right)\right)=0
$$

The implicit equation (45) shows the relationship between $\theta_{A}$ and the friction coefficients $\mu_{d}$ and $\mu_{s}$. If the values of $\mu_{d}$ and $\mu_{s}$ are known, it is possible to calculate the value of $\theta_{A}$.

By substituting Eq. (45) in Eq. (22), and by applying the energy conservation principle between the bottom point and point $A$, it results in,

$$
v_{0}^{2}=\frac{g R}{7}\left(10-17 \cos \left(\theta_{A}\right)+\frac{2}{\mu_{s}} \sin \left(\theta_{A}\right)\right)
$$

Equation (46) provides the minimum velocity at the bottom point of the loop to have a normal force zero at the top. By applying Eq. (2), Eq. (46) results in,

$$
h=\frac{R}{10}\left(10-17 \cos \left(\theta_{A}\right)+\frac{2}{\mu_{s}} \sin \left(\theta_{A}\right)\right)
$$

where $h$ is the minimum initial height of the sphere (see Fig. 1)).

Figure 2 shows the minimum $h(R)$ values against the static friction coefficient under different scenarios

\section{RESULTS AND DISCUSSIONS}

By definition the friction force opposes the slipping motion of one surface on another surface. Thus, the direction of the static friction force is clear. The problem is the dynamic friction force. Between points $A$ and $B$ the sphere slips, so in point $A$, the value of the friction coefficient reduces, changing from the static to the dynamic friction coefficient. This change affects motion equations (10-13). Initially, the absolute value of the angular acceleration decreases, whereas the linear acceleration increases. Therefore, the angular velocity decreases less than the linear velocity $(r \omega>v)$ and the dynamic friction force must have the same behavior than that of the linear velocity, trying to reduce the angular velocity and to increase the linear velocity. Figure 3 shows this idea, displaying the change of the angular velocity compared with the linear velocity for two cases. From Fig. 3 it is possible to understand the evolution of the friction force.

It is noted that when the sphere radius is not much smaller than the loop radius, it is 


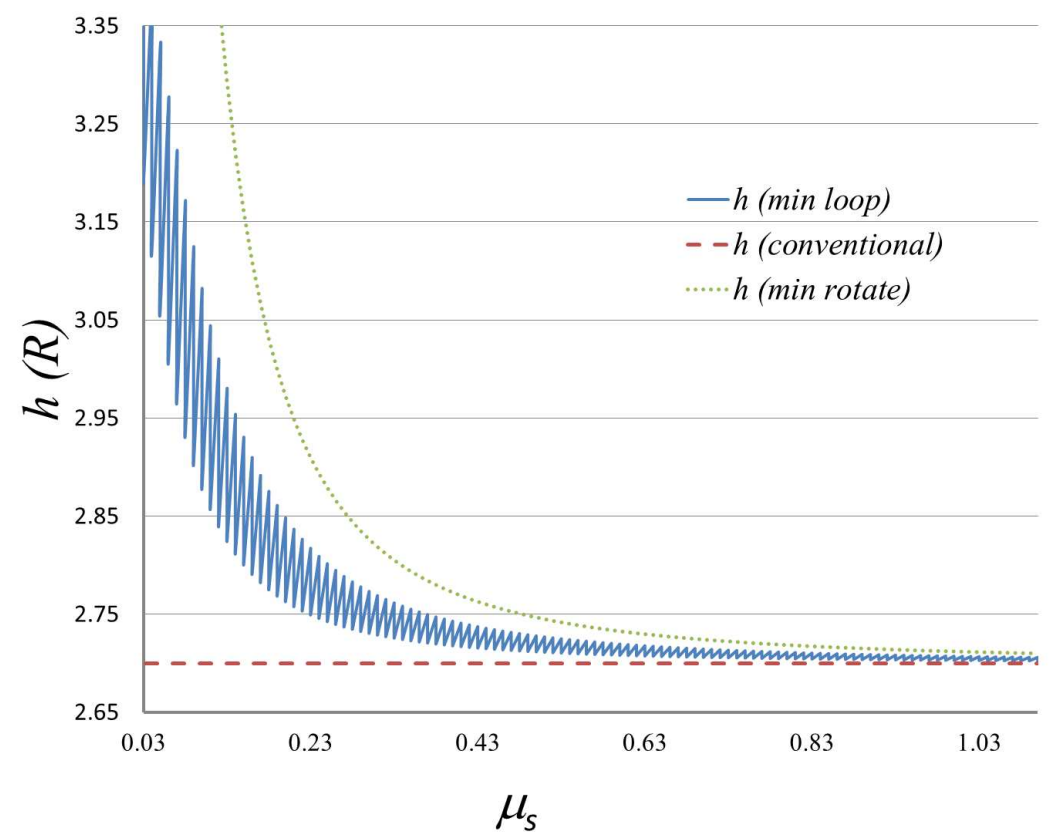

FIG. 2. Normalized minimum initial height of the sphere versus the static friction coefficient. $h$ (min loop): normalized minimum initial height of the sphere to complete a loop, $h$ (min rotate): normalized minimum initial height of the sphere to complete a loop without slipping, $h$ (conventional): normalized minimum initial height of the sphere provided by section II ; against static friction coefficient value

required to replace $R$ by $R-r$ in the solutions given by Eqs. (8), (9), (27), (28), (46) and $(47)$.

\section{CONCLUSIONS}

This paper has proposed a modified formulation of the classical problem of determining the minimum initial height for a sphere that starts from rest, and rolls down an inclined plane, to complete a vertical circular loop in the presence of friction. It has been shown that the two conditions imposed to obtain the classical solution are contradictory, i.e., (1) the speed of the sphere at the top of the loop is that ensuring the normal force to be zero; $(2)$ the sphere must complete the loop without slipping. The solution proposed in this paper has been compared against the solution found in many text books, and the differences found between them have been discussed.

The authors of this article consider that, knowing the skills of first-year undergraduate students, it would be more convenient to use the modified statement proposed in Section III 


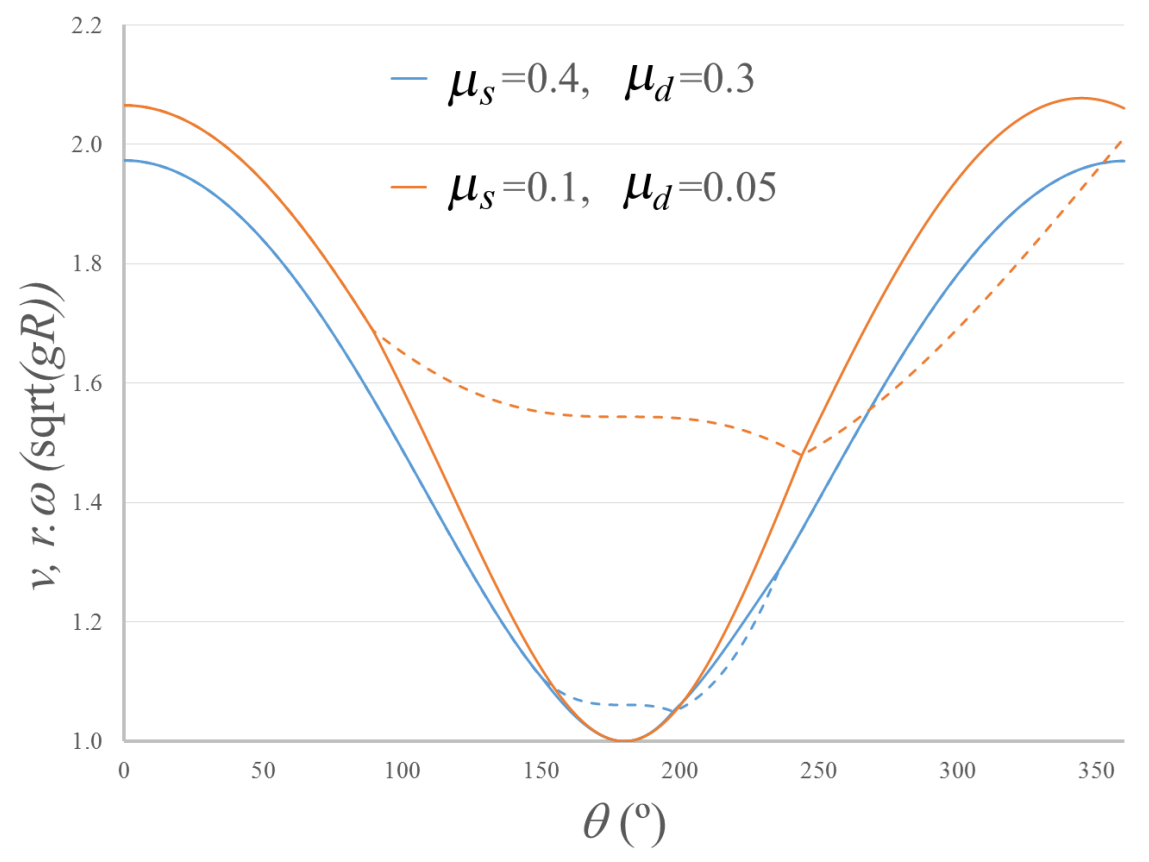

FIG. 3. Evolution of $v$ (solid lines) and $r \omega$ (dashed lines) against $\theta$.

instead of the conventionally one. To solve the problem proposed in Section III, students must have basic knowledge about physics (dynamics, energy conservation and rotational motion) and mathematics (optimization). However, to obtain the exact solution of the conventional problem proposed in Section IV, students require additional knowledge about kinematics and differential equations.

\section{Appendix A: HOW TO CALCULATE THE ROTATIONAL VELOCITY}

At every point of loop path, the motion of the sphere accomplishes (10) and (13)

$$
\begin{aligned}
F_{f}-m g \sin (\theta) & =m a_{t} \\
-F_{f} & =\frac{2}{5} m r \alpha
\end{aligned}
$$

where $F_{f}$ is a friction force. However, this force can be static or dynamic, depending on the analyzed point of the path. The problem is how to calculate the rotational velocity. The velocity and acceleration can be expressed as a function of $\theta$. Since, $a_{t}=\frac{d v}{d t} \quad \alpha=\frac{d \omega}{d t}$ 
By dividing the second relation by the first one, it results in,

$$
\frac{d \omega}{d v}=\frac{\alpha}{a_{t}}
$$

The sum of (A1) and (A2) is as follows,

$$
-m g \sin (\theta)=m a_{t}+\frac{2}{5} m r \alpha
$$

And dividing by $a_{t}$

$$
\begin{aligned}
\frac{r \alpha}{a_{t}} & =-\frac{5}{2}\left(1+\frac{g \sin (\theta)}{a_{t}}\right) \\
\frac{r d \omega}{d v} & =-\frac{5}{2}\left(1+\frac{g \sin (\theta)}{a_{t}}\right) \\
r d \omega & =-\frac{5}{2}\left(1+\frac{g \sin (\theta)}{a_{t}}\right) d v
\end{aligned}
$$

Integrating from one point $\theta_{0}$ (it could be 0 or $A$ ) where we know the rotational velocity

$$
\begin{aligned}
& r \omega=r \omega_{0}-\frac{5}{2}\left(v-v_{0}+\int_{v_{0}}^{v} \frac{g \sin (\theta)}{a_{t}} d v\right) \\
& r \omega=r \omega_{0}-\frac{5}{2}\left(v-v_{0}+\int_{\theta_{0}}^{\theta} \frac{g \sin (\theta)}{v} d \theta\right)
\end{aligned}
$$

By knowing the expression of the velocity, it is possible to determine the rotational velocity. However, from point $A$ to another point between $A$ and $B$, the integral determining the expression of the velocity must be solved numerically. Figure 3 shows (for two pairs of values $\mu_{s}$ and $\mu_{d}$ ) the evolution of the linear velocity (solid lines) and the rotational velocity $r \omega$ (dashed lines) against $\theta$.

\section{ACKNOWLEDGMENTS}

The authors would like to acknowledge Pilar Gil and Jordi Gutierrez, who encouradge us to write this paper. Also, Joan Soler, who always challenges us to solve difficult problems 
and inspired this article.

* oscar.bertran@upc.edu

1 Cina Aghamohammadi and Amir Aghamohammadi, "Slipping and rolling on an inclined plane", Eur. J. Phys., Published 10 June 2011

2 D. E. Shaw and F. J. Wunderlich, "Study of the slipping of a rolling sphere", Am. J. Phys. 52, 997 (1984). https://doi.org/10.1119/1.13915

3 B. Asavapibhop and N. Suwonjandee, "Loop-the-Loop: An Easy Experiment, A Challenging Explanation". AIP Conference Proceedings, 1263, 249 (2010). https://doi.org/10.1063/1. 3479881

4 Waldemar Klobus, "Motion on a vertical loop with friction", Am. J. Phys. 79 (9), 913-918 (2011).

5 Paul A. Tipler and Gene Mosca, Physics. For Scientists and Engineers, 6th edition, Vol. 1 (W. H. Freeman and Company, 2008).

6 N Suwonjandee and B Asavapibhop, "Loop-the-loop: bringing theory into practice", Phys. Educ., 47(6), 751 (2012). http://iopscience.iop.org/0031-9120/47/6/751

7 Peter L. Tea Jr., "Trouble on the loop-the-loop", Am. J. Phys., 55, 826 (1987). https://doi . org/10.1119/1.14997

8 L.P. Franklin and P.I. Kimmel, "Dynamics of circular motion with friction", Am. J. Phys. 48, 207-210 (1980). 